\title{
Surface-Acoustic-Wave Induced Ferromagnetic Resonance in Fe Thin Films and Magnetic Field Sensing
}

\author{
J.-Y. Duquesne $\odot,{ }^{1, *}$ P. Rovillain, ${ }^{1}$ C. Hepburn, ${ }^{1}$ M. Eddrief, ${ }^{1}$ P. Atkinson, ${ }^{1}$ A. Anane,${ }^{2}$ R. Ranchal, ${ }^{3}$ \\ and M. Marangolo ${ }^{1}$ \\ ${ }^{1}$ Sorbonne Université, CNRS, Institut des NanoSciences de Paris, INSP, 4 place Jussieu, F-75005 Paris, France \\ ${ }^{2}$ Unite Mixte de Physique CNRS, Thales, Univ. Paris-Sud, Université Paris Saclay, 1 avenue Augustin Fresnel, \\ F-91767 Palaiseau, France \\ ${ }^{3}$ Universidad Complutense de Madrid, Dpt. Fisica de Materiales. Fac. CC. Fisicas. Plaza de las Ciencias 1, \\ Madrid 28040, Spain
}

(Received 29 January 2019; revised manuscript received 4 June 2019; published 20 August 2019)

\begin{abstract}
Resonant magnetoelastic coupling (MEC) is demonstrated in an Fe thin film epitaxially grown on a piezoelectric GaAs substrate with application of subgigahertz surface acoustic waves (SAWs). The frequency at which resonant MEC is achieved is reduced far below $1 \mathrm{GHz}$ by the application of a small in-plane magnetic field. Moreover, the resonance, observable by attenuation and velocity changes of the SAW, can be switched on and off by a small $\left(0.1^{\circ}\right)$ angular rotation of this in-plane field. This angular sensitivity makes SAW-ferromagnet devices attractive for sensing applications, such as wireless, batteryfree, and interrogable magnetic-field monitors. Using a simple magnetization dynamics model that takes into account the Fe magnetic anisotropy and the softening of the magnetic precession modes, we are able to describe the observed salient features.
\end{abstract}

DOI: 10.1103/PhysRevApplied.12.024042

\section{INTRODUCTION}

Miniaturized magnetic sensors are ubiquitous in modern technology. Most of these devices are based on spintronic effects and, in particular, on magnetoresistance(MR-)related phenomena such as anisotropic, giant, and tunnel magnetoresistance, in which the electrical resistance changes when the direction and the magnitude of an external magnetic field are varied.

Recently, new magnetic field sensors have been proposed that are based on magnetoelastic coupling (MEC) and on the nonresonant interaction between magnetization and acoustic waves [1,2]. This approach is based on mature surface-acoustic-wave (SAW) technology, where hypersounds are used to drive the dynamics of magnetization, because of MEC, rather than dc electrical currents or rf voltages. Like MR-based devices, such magnetic SAW devices are sensitive to the direction and the magnitude of an external magnetic field. SAWs can also be used to develop wireless magnetic field sensors based on coupling a SAW transponder to a giant-magnetoresistance element $[3,4]$.

The main advantage of a strain-mediated approach is that phonons can be excited remotely (and transported over millimeters). They can drive local magnetization dynamics

\footnotetext{
*jean-yves.duquesne@insp.jussieu.fr
}

once they interact with ferromagnetic (FM) materials. This can be done with minimal energy dissipation, very high tunability [5], and high directionality [6]. Moreover, SAW technology is much more appropriate than MR-based technology for addressable, wireless, and battery-free sensors operating in remote or even harsh environments.

Here we consider a SAW-FM device based on resonant MEC (i.e., where the SAW frequency matches the magnetization precession frequency of a ferromagnetic thin film). The physics of resonant coupling between strain and magnetization was pioneered theoretically by Akhiezer et al. [7] and Kittel [8] and experimentally by Bömmel and Dransfeld [9] and Pomerantz [10] many years ago. Later, MEC in the subgigahertz regime allowed SAWinduced ferromagnetic resonance (FMR) to be observed in Ni [11], $(\mathrm{Ga}, \mathrm{Mn})(\mathrm{As}, \mathrm{P})$ [12] and $(\mathrm{Ga}, \mathrm{Mn}) \mathrm{As}$ [13] thin films. Indeed, resonant MEC is so efficient that spinpumping effects could be generated in a $\mathrm{Co} / \mathrm{Pt}$ bilayer at $1.5 \mathrm{GHz}$ [14], and magnetization of $(\mathrm{Ga}, \mathrm{Mn})(\mathrm{As}, \mathrm{P})$ or $(\mathrm{Ga}, \mathrm{Mn}) \mathrm{As}$ thin films could be irreversibly switched [15-17].

There remain some significant challenges in implementing a SAW-induced FMR device: (i) most ferromagnetic materials have precession frequencies well above $5 \mathrm{GHz}$ (i.e., above the highest frequency easily reached by today's SAW technologies); (ii) SAW-based magnetic sensors must prove to be competitive against MR and 
Hall probe devices in accuracy and sensitivity. Additional requirements for materials for future SAW-based spintronic or magnonic devices are that they demonstrate room-temperature SAW-induced FMR, relatively high magnetization for easy detection, a significant MEC, and a low Gilbert damping coefficient, $\alpha$.

In this article, we show that thin films of Fe epitaxially grown on $\mathrm{GaAs}(001)$, a spintronic- and magnoniccompatible [18] magnetoelastic and piezoelectric heterostucture, provide the opportunity to obtain SAW-induced FMR, at room temperature, below $1 \mathrm{GHz}$, despite the high Fe magnetization $\left(M_{s} \simeq 1.7 \times 10^{6} \mathrm{~A} \mathrm{~m}^{-1}\right)$, which is around 3 times larger than in $\mathrm{Ni}$ and 40 times larger than in $(\mathrm{Ga}, \mathrm{Mn})$ As. High saturation magnetization naturally increases the magnetization precession frequency. We show that subgigahertz resonance is obtained when a field is applied in a very tiny angular range $\left(0.1^{\circ}\right)$ with respect to the magnetic hard axis. This angle sensitivity is similar to that of state-of-the-art MR magnetic sensors and Halltechnology-based sensors and permits detection of tiny tilting of the FM magnetization.

\section{GROWTH AND CHARACTERIZATION OF IRON THIN FILMS: DETAILS ON ACOUSTIC MEASUREMENTS}

To obtain high-quality and well-oriented samples, Fe thin films are grown by molecular-beam epitaxy on $\operatorname{GaAs}(001)$ substrates (undoped). Fe thin films are deposited on a $c(2 \times 2) \mathrm{Zn}$-terminated $\mathrm{ZnSe}$ epilayer at $180^{\circ} \mathrm{C}$, grown on a GaAs substrate covered by a thin GaAs buffer, a prototype of a low-reactive iron-semiconductor interface [19]. The ZnSe layer separating the Fe thin film and GaAs substrate ensures an abrupt iron-semiconductor interface [20]. The thickness of the Fe layer is $67 \mathrm{~nm}$. The samples are covered by a protective $10 \mathrm{~nm}$ gold capping layer. The Fe thin films exhibit the expected epitaxial growth relationship that is, $[100]_{\mathrm{Fe}}\left\|[100]_{\mathrm{ZnSe}}\right\|[100]_{\mathrm{GaAs}}$ and $(001)_{\mathrm{Fe}}\left\|(001)_{\mathrm{ZnSe}}\right\|(001)_{\mathrm{GaAs}}$ - and they show biaxial magnetic anisotropy, as reported in previous work [19]. A $4 \times 4 \mathrm{~mm}^{2}$ mesa is then defined by ion etching.

The magnetization of the sample is measured with a vibrating-sample magnetometer [see Fig. 1(a)]. Thin films are characterized by $\mathrm{x}$-ray-diffraction experiments using $\mathrm{Cu} K \alpha$ radiation. The crystalline phases, in-plane orientation, and lattice parameter of the films are evaluated by $\mathrm{x}$-ray diffraction. Typical reciprocal-space maps were collected by measuring $2 \theta / \omega$ radial scan versus $\omega$ rocking scan around the (002), (202), and (112) Bragg reflections of Fe film. Details are given in Ref. [21]. To quantify the mosaicity of the sample, we measure the FWHM of the (002) planes. It is around $0.3^{\circ}$.

Figure 2 presents a sketch of the device under test. We adopted all-electrical excitation and detection of SAWs via the piezoelectric effect in the semi-insulating GaAs
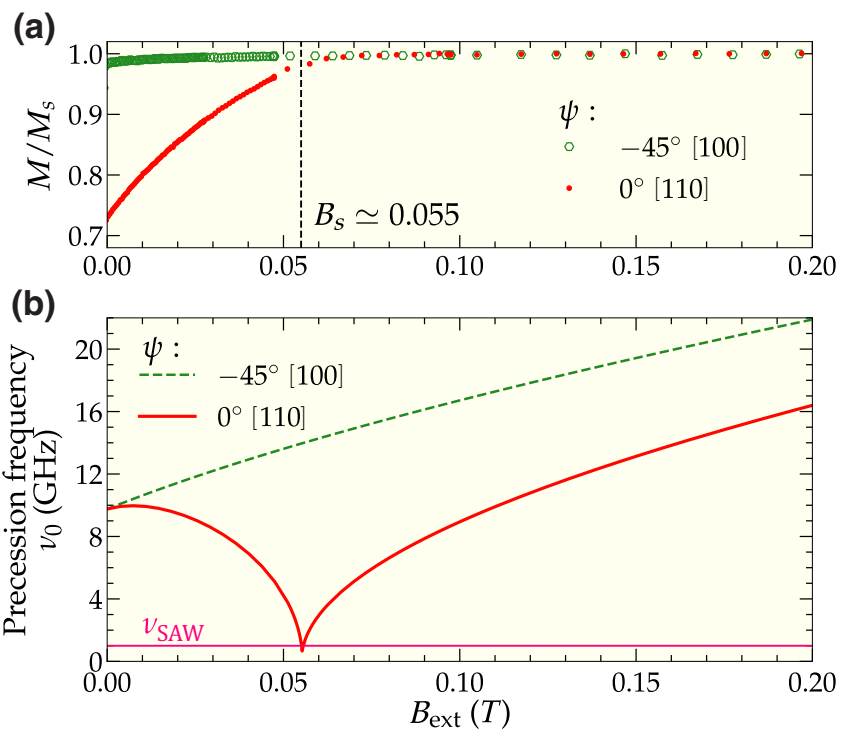

FIG. 1. (a) Magnetization measured along the [100] and [110] axes by a vibrating-sample magnetometer at room temperature. (b) Calculated precession frequencies in a thin Fe layer (inplane magnetization) versus applied field amplitude. The field is applied either along the easy axis [100] $\left(\psi=-45^{\circ}\right)$ or along the hard axis [110] $\left(\psi=0^{\circ}\right)$. The calculation parameters are $M_{s}=1.7 \times 10^{6} \mathrm{~A} \mathrm{~m}^{-1}$, cubic anisotropy $K=4.7 \times 10^{4} \mathrm{~J} \mathrm{~m}^{-3}$, $B_{2}=7 \times 10^{6} \mathrm{~J} \mathrm{~m}^{-3}$ [22], $C_{11}=230 \mathrm{GPa}, C_{12}=134 \mathrm{GPa}$, and $C_{44}=116 \mathrm{GPa}$.

substrate [23] using interdigitated transducers (IDTs) [24]. The emitter-IDT orientation is such that the acoustic wave vector $\mathbf{k}_{\mathrm{SAW}}$ is parallel to [110], the most-favorable direction for SAW excitation in GaAs. The "split-44" design [25] is used to excite the SAW at four harmonic frequencies $n v$, where $v=119 \mathrm{MHz}$ and $n=1,3,5,7$. Pulsed excitation is used with 500-ns duration. An identical IDT acts as a detector. The $\mathbf{k}_{\mathrm{SAW}}$ direction can be reversed by connecting the input signal to one IDT or to the other. The sample is first subjected to a large external reference field $\mathbf{B}_{\text {ext }}$, the magnitude of which is $B_{\text {ref }}=0.4 \mathrm{~T}$ and the direction of which is at an angle $\psi$ to [110]. This large external field saturates the sample. The field intensity is then decreased to zero while the

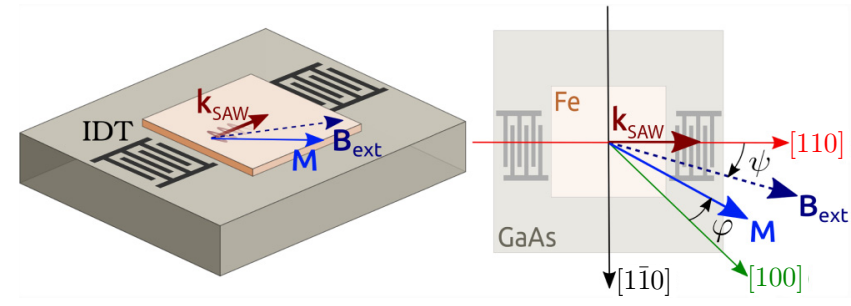

FIG. 2. Sketch of the sample: $4 \times 4 \mathrm{~mm}^{2}$ Fe mesa $(67 \mathrm{~nm})$ on $\mathrm{GaAs}(001)$. The IDT has a split-44 design [25]. The wave vector $\mathbf{k}_{\mathrm{SAW}}$ is parallel to [110], $\mathbf{B}_{\mathrm{ext}}$ is the in-plane applied magnetic field, and $\mathbf{M}$ is the Fe magnetization. 
amplitude and phase of the acoustic signal are measured. We deduce the relative change in SAW phase velocity, $\Delta V / V=\left[V\left(B_{\text {ext }}\right)-V\left(B_{\text {ref }}\right)\right] / V\left(B_{\text {ref }}\right)$, and the change in SAW attenuation, $\Delta \Gamma=\Gamma\left(B_{\text {ext }}\right)-\Gamma\left(B_{\text {ref }}\right)$, as a function of the field amplitude $B_{\text {ext }}$ at a given field direction $\psi$ and at a given acoustic frequency $\nu_{\mathrm{SAW}}$. We recall that the acoustic attenuation $\Gamma$, in decibels per unit length (centimeters), is $\Gamma=(20 / \ln 10) L^{-1}$, where $L$ characterizes the exponential decay $\exp (-x / L)$ of the acoustic wave amplitude versus propagation distance $x$. All experiments are performed at room temperature.

\section{RESULTS AND DISCUSSION}

\section{A. Evidence of subgigahertz resonance and angle sensitivity in epitaxially grown iron thin films}

As shown in Fig. 1(b) (see the dashed green line) the precession frequency of $\mathrm{Fe}$ single crystals, $v_{0}$, calculated with the approach of Smit and Beljer [26] is $10 \mathrm{GHz}$ at zero field due to the high biaxial magnetic anisotropy constant $K=4.7 \times 10^{4} \mathrm{~J} \mathrm{~m}^{-3}$. It can be seen that $\nu_{0}$ increases with increasing $M_{s} B_{\text {ext }}$ when an external field $B_{\text {ext }}$ is applied along the easy axis [100]. Such high frequencies restrict research on SAW-induced FMR to low-magnetization and/or low-magnetic-anisotropy materials, such as $(\mathrm{Ga}, \mathrm{Mn}) \mathrm{As}$ or $(\mathrm{GaMn})(\mathrm{As}, \mathrm{P})[12,13,15,16]$, Ni $[6,9,11,14,27-29]$, and yttrium iron garnet [30,31].

Here we propose to lower $\nu_{0}$ below the gigahertz regime by applying $\mathbf{B}_{\text {ext }}$ along the in-plane hard axis of $\mathrm{Fe}$; namely, the [110] and [110] directions. This strategy of applying $\mathbf{B}_{\mathrm{ext}}$ to lower $\nu_{0}$ was used previously for $(\mathrm{Ga}, \mathrm{Mn})(\mathrm{As}, \mathrm{P})$ [12]. It may also have lowered $\nu_{0}$ in polycrystalline Co to $1.5 \mathrm{GHz}$ [14] (not discussed in Ref. [14]). In this configuration, the approach of Smit and Beljer predicts a softening of the effective magnetic stiffness at the saturation field $\left[B_{s} \simeq 55 \mathrm{mT}\right.$; see the experimental magnetization in Fig. 1(a)]. As shown in Figs. 1(b) and 3, at $B_{s}$, the precession frequency $v_{0}$ decreases to a value close to typical SAW frequencies, permitting resonant MEC [32]. In the absence of coupling, $v_{0}$ is zero. The effect of the magnetoelastic coupling is to increase the minimum value of $v_{0}$. Readers interested in the full calculation can find details in Ref. [33]. Such a softening is extremely angular dependent as shown in Fig. 3: $v_{0}$ increases dramatically when the magnetic field direction is only $1^{\circ}$ off the [110] direction or the [1 10$]$ direction. For this reason, highly oriented Fe crystals are required to get resonant MEC at subgigahertz frequencies.

Figures 4 and 5 display our main results. We observe attenuation peaks with a maximum amplitude when $\mathbf{B}_{\text {ext }}$ is parallel, within the experimental accuracy of $\pm 1^{\circ}$, to the in-plane hard axis [110] $\left(\psi=0^{\circ}\right)$. Figure 4(a) shows the strong dependence of the SAW attenuation peak on the angle of the applied in-plane field at $833 \mathrm{MHz}$. The variation in attenuation with external in-plane magnetic

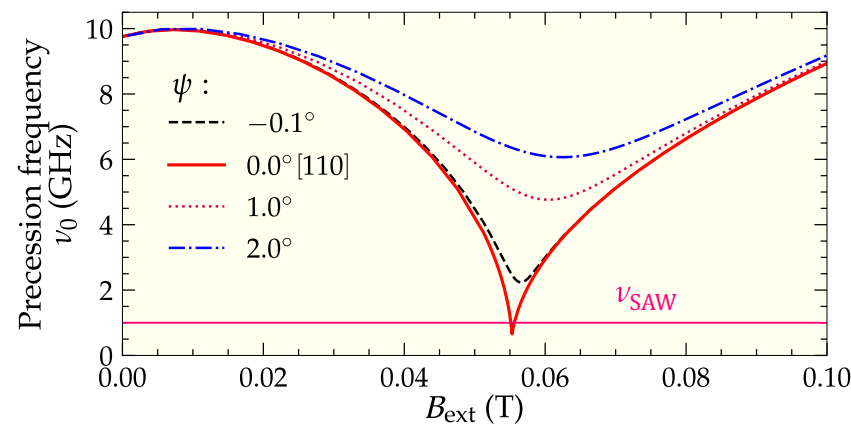

FIG. 3. Calculated precession frequencies for various small applied-field angles $\psi$ with respect to [110]. For the calculation parameters, see Fig. 1(b).

field is rather large $\left(5 \mathrm{~dB} \mathrm{~cm}^{-1}\right)$, and maximum attenuation is obtained for $0.055 \mathrm{~T}$ along the [110] direction, which corresponds to the saturation field [Fig. 1(a)] and to the minimum of the magnetization precession frequency [Figs. 1(b) and 3]. The very strong angular dependence of the effect is a striking result: rotating the field from $0.0^{\circ}$ to $0.1^{\circ}$ reduces the attenuation peak by a factor of 5. Consequently, the experiments require an experimental resolution of at least $0.05^{\circ}$. It can also be noted that the magnitude of the attenuation peak depends on whether the acoustic wave vector $\mathbf{k}_{\mathrm{SAW}}$ is parallel or antiparallel to the applied field $\mathbf{B}_{\text {ext }}$, as shown in the inset in Fig. 4(a).
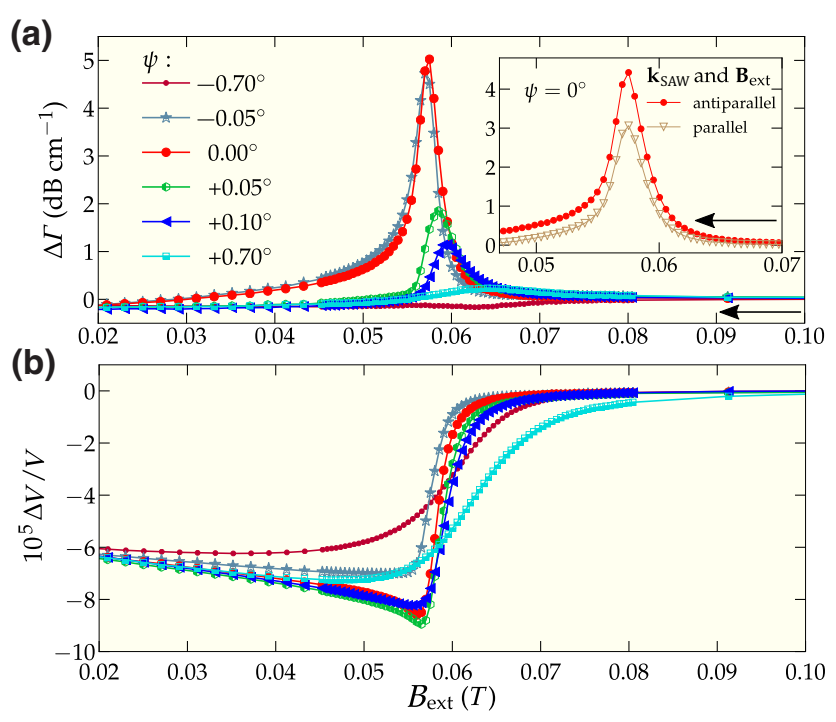

FIG. 4. Variation of (a) acoustic attenuation $\Gamma$ and (b) velocity $V$ versus applied-magnetic-field amplitude $B_{\text {ext }}$ at various angles $\psi$. The applied field $\mathbf{B}_{\text {ext }}$ is in the plane at an angle $\psi$ with respect to the [110] direction (hard axis). Measurements are taken as the field amplitude is decreasing. $\Delta \Gamma=\Gamma\left(B_{\text {ext }}\right)-\Gamma\left(B_{\text {ref }}\right), \Delta V / V=$ $\left[V\left(B_{\text {ext }}\right)-V\left(B_{\text {ref }}\right)\right] / V\left(B_{\text {ref }}\right)$, and $B_{\text {ref }}=0.4 \mathrm{~T}$. The acoustic frequency is $833 \mathrm{MHz}$. The SAW wave vector $\mathbf{k}_{\mathrm{SAW}}$ is parallel to the [110] direction and antiparallel to $\mathbf{B}_{\text {ext }}$. In the inset in (a) the magnetic configuration is the same, $\psi=0^{\circ}: \mathbf{k}_{\mathrm{SAW}}$ is either parallel or antiparallel to $\mathbf{B}_{\mathrm{ext}}$. 
(a)
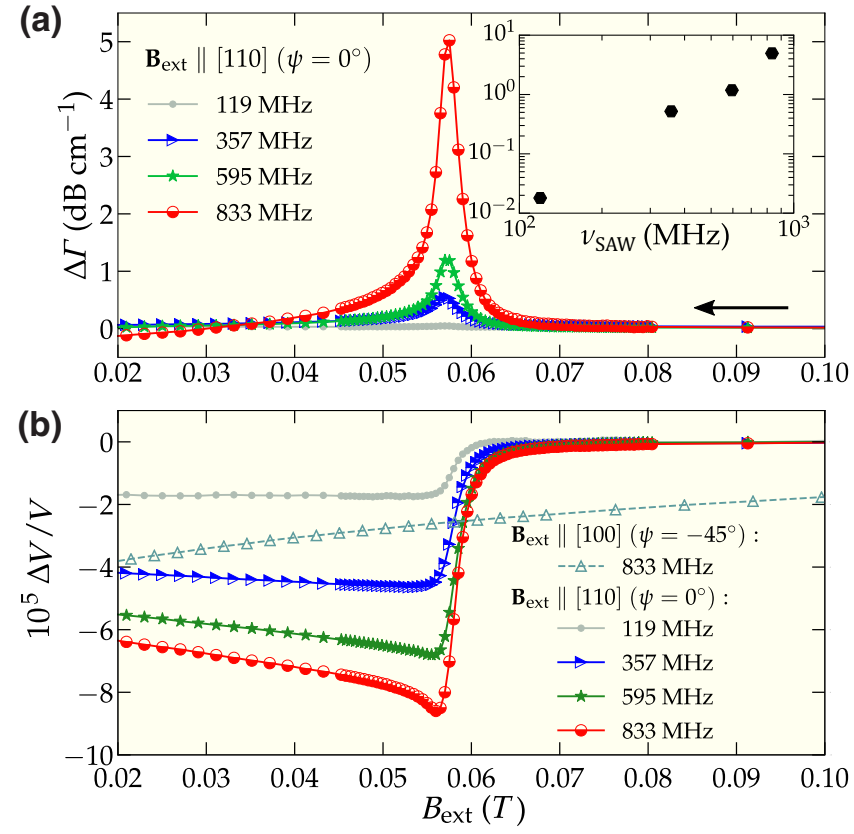

FIG. 5. Variation of (a) acoustic attenuation $\Gamma$ and (b) velocity $V$ versus applied-magnetic-field amplitude $B_{\text {ext }}$ at various frequencies. The applied field $\mathbf{B}_{\mathrm{ext}}$ is in the plane parallel to the [110] direction (hard axis, $\psi=0^{\circ}$ ) or to the [100] direction (easy axis, $\psi=-45^{\circ}$ ). The field amplitude is decreasing. $\Delta \Gamma=\Gamma\left(B_{\text {ext }}\right)-\Gamma\left(B_{\text {ref }}\right), \quad \Delta V / V=\left[V\left(B_{\text {ext }}\right)-V\left(B_{\text {ref }}\right)\right] / V\left(B_{\text {ref }}\right)$, and $B_{\text {ref }}=0.4 \mathrm{~T}$. The acoustic frequency ranged from 119 to 833 MHz. $\mathbf{k}_{\mathrm{SAW}}$ is parallel to the [110] direction. At $\psi=0^{\circ}, \mathbf{k}_{\mathrm{SAW}}$ and $\mathbf{B}_{\text {ext }}$ are antiparallel. The inset in (a) shows the magnitude of the attenuation peak versus frequency $\left(\psi=0^{\circ}\right)$.

Such nonreciprocity effects are well known and are frequently observed in magnetic systems [34-36]. In the following we consider only the antiparallel configuration, where the attenuation peak is slightly larger. Figure 4(b) shows the velocity variation versus applied field for various angles $\psi$. Steep variations are observed around the saturating field, with a kink at $0.055 \mathrm{~T}$ for angles $\psi$ close to zero.

To evidence the effect of the SAW-induced FMR on the experimental curves, we compare in Fig. 5 both SAWattenuation $\Delta \Gamma$ and phase-velocity variations $\Delta V / V$ at several acoustic frequencies $\nu_{\mathrm{SAW}}$ and for two magnetic field directions. We first consider $\mathbf{B}_{\text {ext }}$ parallel to the inplane hard axis [110] $\left(\psi=0^{\circ}\right)$. At high external field, both attenuation and velocity are constant. Below 0.08 $\mathrm{T}$, we observe velocity and attenuation variations indicating an effective magnetoelastic coupling with a maximum close to the saturation field $B_{s}$. In the inset in Fig. 5(a), we show that the magnitude of the attenuation peak is roughly proportional to $v_{\mathrm{SAW}}^{3}$. Moreover, the velocity change depends on the frequency. It is also worthwhile noticing in Fig. 5(b) the kink in the velocity variations at $833 \mathrm{MHz}$ around $0.055 \mathrm{~T}$, which fades away when the SAW frequency decreases. A totally different behavior is observed when the field is applied along the [100] direction $\left(\psi=-45^{\circ}\right)$. The attenuation is constant (not shown) and the velocity changes smoothly up to high fields [see Fig. 5(b)].

\section{B. Phenomenological model}

To evaluate how resonant MEC affects the field dependence of velocity and attenuation, we study magnetization dynamics in the framework of the Landau-Lifshitz-Gilbert equation for a uniform mode:

$$
\frac{d \mathbf{m}}{d t}=-\gamma \mathbf{m} \times \mathbf{B}_{\mathrm{eff}}+\alpha \mathbf{m} \times \frac{d \mathbf{m}}{d t}, \quad \mathbf{B}_{\mathrm{eff}}=-\nabla_{\mathbf{m}} f,
$$

where $\mathbf{m}$ is the normalized magnetization $\mathbf{M} / M_{s}, \gamma$ is the absolute value of the gyromagnetic factor, and $\alpha$ is the Gilbert damping coefficient. For simplicity, we assume that the damping is fully described by $\alpha . f$ is the normalized free energy $F / M_{s}$ of the layer. The free energy $F$ is the sum $F=F_{Z}+F_{d}+F_{\mathrm{mc}}+F_{\mathrm{me}}+F_{\mathrm{el}}$, where the various terms are the Zeeman, the demagnetizing, the magnetocrystalline-anisotropy, the magnetoelastic, and the elastic energy terms. The relative weight of each term depends on the intensity and the direction of the external magnetic field (see, e.g., Ref. [33]). The magnetoelastic free energy of a cubic solid reads

$$
\begin{aligned}
F_{\mathrm{me}}= & B_{1}\left[\varepsilon_{x x}\left(m_{x}^{2}-\frac{1}{3}\right)+\varepsilon_{y y}\left(m_{y}^{2}-\frac{1}{3}\right)+\varepsilon_{z z}\left(m_{z}^{2}-\frac{1}{3}\right)\right] \\
& +2 B_{2}\left(\varepsilon_{x y} m_{x} m_{y}+\varepsilon_{x z} m_{x} m_{z}+\varepsilon_{y z} m_{y} m_{z}\right)
\end{aligned}
$$

where $B_{1}$ and $B_{2}$ are phenomenological coupling constants [37], and $\varepsilon_{i j}$ are the strain components, expressed in the standard cubic frame. For small deviations from equilibrium, the Landau-Lifshitz-Gilbert equation can be linearized $[33,38]$ :

$$
\begin{aligned}
& \frac{d \delta \varphi}{d t}=\kappa_{1} \delta \theta+\kappa_{2} \delta \varphi+\sum_{i \leq j} \kappa_{i j} \delta \varepsilon_{i j}, \\
& \frac{d \delta \theta}{d t}=\zeta_{1} \delta \theta+\zeta_{2} \delta \varphi+\sum_{i \leq j} \zeta_{i j} \delta \varepsilon_{i j},
\end{aligned}
$$

where $\theta$ and $\varphi$ are the magnetization $\mathbf{M}$ polar and azimuthal angles (with respect to [001] and [100], respectively). The $\kappa$ and $\zeta$ terms are related to the second-order derivatives $f_{\theta, \theta}, f_{\varphi, \varphi}$, and $f_{\theta, \varphi}$ of the normalized free energy (for details, see Ref. [33]). In our experiments, the deviations $\delta \varepsilon_{i j}$ are due to the surface acoustic wave. To simplify the calculations, we crudely assume that a bulk longitudinal wave, propagating along the [110] direction may catch the physics of the interaction between the acoustic wave and M. Such a simple model has already been 
used [39]. More-sophisticated calculations would certainly improve the agreement with the experimental data but at the expense of complexity [40-42].

The displacement-field $\mathbf{u}$ and precession-angle variations $\delta \varphi$ and $\delta \theta$ read

$$
\begin{aligned}
& u_{1}^{\prime}=U \exp \left[i\left(\omega t-k x^{\prime}\right)\right], \\
& u_{2}^{\prime}=u_{3}^{\prime}=0, \\
& \delta \varphi=\Phi \exp \left[i\left(\omega t-k x^{\prime}\right)\right], \\
& \delta \theta=\Theta \exp \left[i\left(\omega t-k x^{\prime}\right)\right],
\end{aligned}
$$

where here, and in the following, any component expressed in the rotated frame $([110],[\overline{1} 10],[001])$ is primed. From Eqs. (3) and (4), we derive

$$
\delta \varphi=\frac{-i k}{2} \frac{\left(i \omega-\zeta_{1}\right) \kappa_{12}+\zeta_{12} \kappa_{1}}{\left(i \omega-\kappa_{2}\right)\left(i \omega-\zeta_{1}\right)-\kappa_{1} \zeta_{2}} u_{1}^{\prime}
$$

The acoustic wave dynamics is governed by the Newton equation. In our case

$$
\rho \frac{\partial^{2} u_{1}^{\prime}}{\partial t^{2}}=\frac{\partial \sigma_{11}^{\prime}}{\partial x^{\prime}}
$$

where $\rho$ is the mass density of the magnetic layer, and the strain $\sigma_{11}^{\prime}=\partial F / \partial \varepsilon_{11}^{\prime}$ is derived from the magnetic free energy $F$, where

$$
\sigma_{11}^{\prime}=C_{11}^{\prime} \delta \varepsilon_{11}^{\prime}+B_{2} \cos 2 \bar{\varphi} \delta \varphi,
$$

where $\bar{\varphi}$ is the equilibrium value of the azimuthal angle $\varphi$. The relation between $\bar{\varphi}$ and $\psi$ can be derived by a Stonerand-Wohlfarth approach by minimizing the free energy. At saturation, $\psi$ and $\bar{\varphi}$ are linked by $\psi=\bar{\varphi}-\pi / 4$. $C_{11}^{\prime}$ is the $C_{11}$ elastic constant of the Fe layer in the rotated frame ([110], [110], [001]). The magnetoelastic term in Eq. (7) reflects the maximum torque exerted by the SAW on the magnetization for $\bar{\varphi}=0^{\circ}$ or $90^{\circ}$. In contrast, for $\bar{\varphi}=45^{\circ}$ [i.e., when a saturating field is applied along the [110] direction $\left(\psi=0^{\circ}\right)$ ] the coupling vanishes. This is consistent with the experimental results: no attenuation or velocity changes versus $B_{\text {ext }}>0.08 \mathrm{~T}$ are observed in this configuration (see Fig. 5). From Eqs. (5)-(7), we derive

$$
\rho \omega^{2}=\widetilde{C_{11}^{\prime}} k^{2},
$$

where

$$
\widetilde{C_{11}^{\prime}}=C_{11}^{\prime}+\frac{B_{2}^{2} \gamma}{M_{s}} \cos ^{2} 2 \bar{\varphi} \frac{\left(\omega^{2}-\omega_{0}^{2}\right) \gamma f_{\theta, \theta}-2 \alpha \omega^{2} \tau^{-1}+i\left[2 \omega \tau^{-1} \gamma f_{\theta, \theta}+\alpha \omega\left(\omega^{2}-\omega_{0}^{2}\right)\right]}{\left(\omega^{2}-\omega_{0}^{2}\right)^{2}+4 \omega^{2} \tau^{-2}},
$$

where $\tau$ is the relaxation time and $\omega_{0}=2 \pi v_{0}$ is the magnetization-precession resonant radial frequency, which are defined in Ref. [33] as

$$
\begin{aligned}
\tau^{-1} & =\frac{1}{2} \frac{\alpha \gamma}{1+\alpha^{2}}\left(f_{\theta, \theta}+f_{\varphi, \varphi}\right), \\
\omega_{0} & =\gamma \sqrt{f_{\theta, \theta} f_{\varphi, \varphi}} .
\end{aligned}
$$

$\widetilde{C_{11}^{\prime}}$ is an effective elastic modulus governing the propagation of a bulk longitudinal wave along the [110] direction in an infinite medium with elastic and magnetic properties of the layer. We take into account the finite thickness $d$ of the thin film by an effective-medium approach. The penetration depth of a SAW is roughly equal to its wavelength $\lambda$ (in our sample $\lambda \simeq 3.4 \mu \mathrm{m}$ at $833 \mathrm{MHz}$ and $d=67 \mathrm{~nm}$ ). We assume that the relevant effective modulus, governing the propagation of the SAW, is the average of the layer and substrate modulus weighted by $d$ and $(\lambda-d)$, respectively:

$$
C^{\prime}=\frac{d \widetilde{C_{11}^{\prime}}+(\lambda-d) C_{s}^{\prime}}{\lambda}
$$

where $C_{s}^{\prime}$ is the $C_{11}$ elastic modulus of the substrate in the rotated frame. The velocity $v$ and acoustic decay length $L$ are related to the real and imaginary parts of $C^{\prime}$ [43]:

$$
\frac{\Delta v}{v}=\frac{d}{2 \lambda} \frac{\Delta \operatorname{Re} \widetilde{C_{11}^{\prime}}}{C_{s}^{\prime}}, \quad L^{-1}=\frac{\omega^{2} d}{4 \pi v^{2}} \frac{\operatorname{Im} \widetilde{C_{11}^{\prime}}}{C_{s}^{\prime}}
$$

We now show that this simple model catches the physics of the observed phenomenon (i.e., a highly directional resonant process). As discussed above, magnetoelastic resonance can be achieved close to $B_{s}$, as shown in Fig. 3 . Careful inspection of Eqs. (9) and (13) reveals the resonant character of $\widetilde{C_{11}^{\prime}}$ with a maximum when $\omega_{0}$ and $\omega_{\text {SAW }}$ match, leading to maximum attenuation and velocity variations. This is visible in Fig. 6(a), which shows the calculated attenuation for several small angles $\psi$ with respect to the [110] direction at $833 \mathrm{MHz}$.

Our simple model predicts attenuation peaks exhibiting a strong dependence on $\psi$, in agreement with the experimental results. This is related to the sensitivity of the frequency-matching condition with respect to $\psi$ (see 
(a)

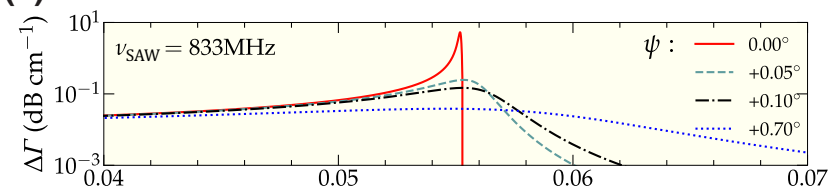

(b)

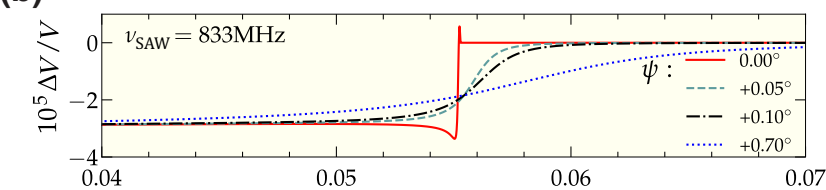

(c)

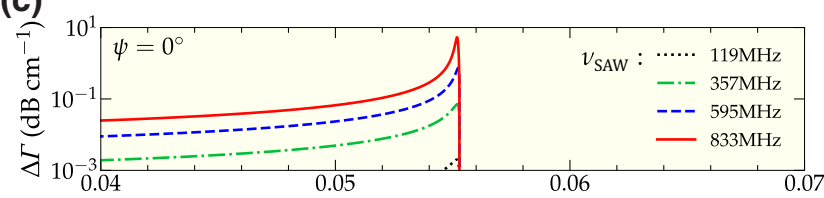

(d)

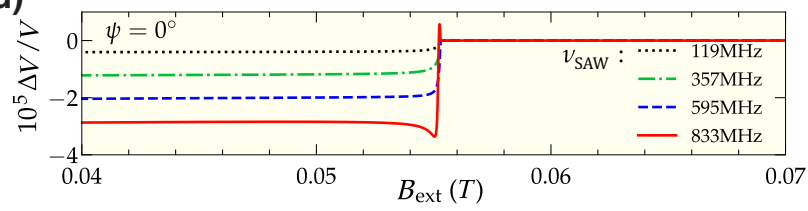

FIG. 6. Computed (a),(c) acoustic attenuation $\Gamma$ and (b),(d) velocity $V$ changes versus amplitude $B_{\text {ext }}$ of the applied field. The calculation parameters are the same as those for Fig. 1(b) and $\alpha=0.005$. The applied field $\mathbf{B}_{\text {ext }}$ is in the (001) plane at an angle $\psi$ from the [110] direction (hard axis). The field amplitude is decreasing. (a),(b) Attenuation and velocity changes at several small angles $\psi$ off [110] at $833 \mathrm{MHz}$. (c),(d) Attenuation and velocity changes at several acoustic frequencies from 119 to 833 $\mathrm{MHz}$ at $\psi=0^{\circ}$.

Fig. 3). Figure 6(c) shows the attenuation at various frequencies at $\psi=0^{\circ}$. At $833 \mathrm{MHz}$, the attenuation is much larger than at lower frequencies due to the matching condition $\omega_{\mathrm{SAW}}=\omega_{0}$. The magnitude of the calculated peak is close to the experimental value $\left(5 \mathrm{~dB} \mathrm{~cm}^{-1}\right)$ and decreases drastically at lower frequencies. The experimental curves are wider than the theoretical ones. We ascribe this observation to the mosaicity of the sample [the FWHM of the (002) planes is approximately $0.3^{\circ}$, measured by $\mathrm{x}$ ray diffraction], which is responsible for spreading of the resonant frequencies at a given $\mathbf{B}_{\text {ext }}$. At low frequencies, the calculation underestimates the coupling since the measured attenuation is slightly larger than that predicted by our simple model. This could be due to an underestimation of the magnetic losses at low frequencies due to the contribution of the inhomogeneous broadening of the FMR linewidth. Otherwise, our calculations show that the velocity changes are quite insensitive to the damping term $\alpha$. The resonant frequency of devices based on mechanical resonance will also be quite insensitive to the damping term $\alpha$ even if the width of the resonance is affected by $\alpha$.

With regard to sound velocity, our calculation gives the correct order of magnitude and describes the trend of the magnetic field dependence as reported in Figs. 6(b) and 6(d). A sharp variation is observed at all frequencies for $B_{\text {ext }}=B_{s}$. At $833 \mathrm{MHz}$ and $\psi=0$, we notice that the variation is maximum and it is characterized by two kinks just above and below the sharp step. These kinks are the fingerprint of the resonant process. They are intimately related to the crossing of the $\omega_{0}\left(B_{\text {ext }}\right)$ and $\omega_{\text {SAW }}$ curves (see Fig. 3) and to the change of sign of the $\omega_{0}^{2}-\omega_{\text {SAW }}^{2}$ term in Eq. (9) when $B_{\text {ext }}$ is swept. This can easily be understood assuming $\alpha=0$ in Eq. (9). In that case, $\widetilde{C_{11}^{\prime}}$ diverges at $\omega_{\mathrm{SAW}}=\omega_{0}$ but with positive sign if $\omega_{0} \rightarrow \omega_{\mathrm{SAW}}^{-}$(i.e., if $B_{\text {ext }}$ decreases, starting form $B_{s}$ ) and negative sign if $\omega_{0} \rightarrow \omega_{\text {SAW }}^{+}$(i.e., if $B_{\text {ext }}$ increases, starting from 0 ). When the damping term $\alpha$ is taken into account, the divergence is replaced by large positive (upper kink) and negative (lower kink) variations [Figs. 6(b) and 6(d)]. The wider (lower) kink reproduces well the kink observed in experiments at $833 \mathrm{MHz}$ and $\psi=0$ [Figs. 4(b) and 5(b)], indicating that the condition $\omega_{\mathrm{SAW}}=\omega_{0}$ is experimentally fulfilled. Our calculations indicate that the narrower (upper) kink is not observed experimentally because of a convolution effect due to the mosaicity of the sample (not shown). Finally, the $\omega_{\text {SAW }}=\omega_{0}$ condition is certainly fulfilled even at 595 $\mathrm{MHz}$ since we observe a small kink below the sharp step at $595 \mathrm{MHz}$ [see Fig. 5(b)].

\section{CONCLUSION AND PERSPECTIVES}

Our experimental findings prove that resonant phononmagnon coupling can be comfortably obtained in epitaxial Fe thin films on piezoelectric GaAs(001), even in the subgigahertz regime.

We show experimentally and confirm by a simple phenomenological model that application of an in-plane field can bring the thin-film magnetization precession frequency into resonance with the applied SAW. The subsequent attenuation and variation in velocity of the SAW is highly sensitive to both the orientation and the magnitude of this applied in-plane field, in good agreement with the dependence of the thin-film magnetization frequency on the applied in-plane field. In particular, the experimentally observed kink in the SAW-velocity variation for SAW frequencies of 833 and $595 \mathrm{MHz}$ is predicted by our model and is a direct indicator of the matching condition $\omega_{\text {SAW }}=$ $\omega_{0}$. These frequencies are fully compatible with today's SAW technology.

We show that SAW-induced FMR is extremely sensitive to the angle of the applied in-plane magnetic field. Indeed, the magnetization dynamics can be switched on or off by an approximately $0.1^{\circ}$ rotation of the magnetic field direction. Consequently, our device can detect a magnetic field of approximately $0.1 \mathrm{mT}$ directed perpendicular to the $55-\mathrm{mT}$ bias field. This sensitivity is competitive with existing MR-based magnetic field sensors, with the advantage that SAW-based devices allow wireless and autonomous 
sensing. Our experiments are performed on Fe thin films grown by molecular-beam epitaxy. However, other FM materials and other growth techniques could be valuable, provided the materials exhibit a large anisotropy, induced either by the magnetocrystalline field or by the growth process [44]. $\mathrm{Fe}_{1-x} \mathrm{Ga}_{x}$ and $\mathrm{Ni}-\mathrm{Fe}$ (permalloy) are such materials.

\section{ACKNOWLEDGMENTS}

R.R. acknowledges financial support through the project MAT2015-66888-C3-3-R (MINECO/FEDER) of the Spanish Ministry of Economy and Competitiveness. The authors thank C. Gourdon and L. Thevenard for fruitful discussions and careful reading of the manuscript. They acknowledge the staff of the MPBT (physical properties - low temperature) platform of Sorbonne University for their support as well as L. Becerra and M. Rosticher for optical and electronic lithography.

[1] V. Polewczyk, K. Dumesnil, D. Lacour, M. Moutaouekkil, H. Mjahed, N. Tiercelin, S. Petit Watelot, H. Mishra, Y. Dusch, S. Hage-Ali, O. Elmazria, F. Montaigne, A. Talbi, O. Bou Matar, and M. Hehn, Unipolar and Bipolar HighMagnetic-Field Sensors Based on Surface Acoustic Wave Resonators, Phys. Rev. Appl. 8, 024001 (2017).

[2] M. Elhosni, O. Elmazria, S. Petit-Watelot, L. Bouvot, S. Zhgoon, A. Talbi, M. Hehn, K. A. Aissa, S. Hage-Ali, D. Lacour, F. Sarry, and O. Boumatar, Magnetic field SAW sensors based on magnetostrictive-piezoelectric layered structures: FEM modeling and experimental validation, Sens. Actuators A: Phys. 240, 41 (2016).

[3] H. Al Rowais, B. Li, C. Liang, S. Green, Y. Gianchandani, and J. Kosel, Development of a passive and remote magnetic microsensor with thin-film giant magnetoimpedance element and surface acoustic wave transponder, J. Appl. Phys. 109, 07E524 (2011).

[4] P. Zhang, H. Hu, F. Muhammad, and Y. Lei, High frequency passive micro-magnetic sensor based on surface acoustic wave transponder and giant magnetoimpedance sensitive element, Sens. Actuators A: Phys. 254, 54 (2017).

[5] D. Labanowski, A. Jung, and S. Salahuddin, Power absorption in acoustically driven ferromagnetic resonance, Appl. Phys. Lett. 108, 022905 (2016).

[6] P. G. Gowtham, T. Moriyama, D. C. Ralph, and R. A. Buhrman, Traveling surface spin-wave resonance spectroscopy using surface acoustic waves, J. Appl. Phys. 118, 233910 (2015).

[7] A. Akhiezer, V. Bariakhtar, and S. Peletminskii, Coupled magnetoelastic waves in ferromagnetic media and ferroacoustic resonance, Sov. Phys. JETP-USSR 8, 157 (1959).

[8] C. Kittel, Interaction of spin waves and ultrasonic waves in ferromagnetic crystals, Phys. Rev. 110, 836 (1958).

[9] H. Bömmel and K. Dransfeld, Excitation of Hypersonic Waves by Ferromagnetic Resonance, Phys. Rev. Lett. 3, 83 (1959).
[10] M. Pomerantz, Excitation of Spin-wave Resonance by Microwave Phonons, Phys. Rev. Lett. 7, 312 (1961).

[11] M. Weiler, L. Dreher, C. Heeg, H. Huebl, R. Gross, M. S. Brandt, and S. T. B. Goennenwein, Elastically Driven Ferromagnetic Resonance in Nickel Thin Films, Phys. Rev. Lett. 106, 117601 (2011).

[12] L. Thevenard, C. Gourdon, J. Y. Prieur, H. J. von Bardeleben, S. Vincent, L. Becerra, L. Largeau, and J.Y. Duquesne, Surface-acoustic-wave-driven ferromagnetic resonance in $(\mathrm{Ga}, \mathrm{Mn})(\mathrm{As}, \mathrm{P})$ epilayers, Phys. Rev. B 90, 094401 (2014).

[13] P. Kuszewski, J.-Y. Duquesne, L. Becerra, A. Lemaître, S. Vincent, S. Majrab, F. Margaillan, C. Gourdon, and L. Thevenard, Optical Probing of Rayleigh Wave Driven Magnetoacoustic Resonance, Phys. Rev. Appl. 10, 034036 (2018).

[14] M. Weiler, H. Huebl, F. S. Goerg, F. D. Czeschka, R. Gross, and S. T. B. Goennenwein, Spin Pumping with Coherent Elastic Waves, Phys. Rev. Lett. 108, 176601 (2012).

[15] L. Thevenard, I. S. Camara, S. Majrab, M. Bernard, P. Rovillain, A. Lemaître, C. Gourdon, and J.-Y. Duquesne, Precessional magnetization switching by a surface acoustic wave, Phys. Rev. B 93, 134430 (2016).

[16] P. Kuszewski, I. Camara, N. Biarrotte, L. Becerra, J. von Bardeleben, W. Savero Torres, A. Lemaître, C. Gourdon, J.-Y. Duquesne, and L. Thevenard, Resonant magnetoacoustic switching: Influence of Rayleigh wave frequency and wavevector, J. Phys.: Condens. Matter 30, 244003 (2018).

[17] I. S. Camara, J.-Y. Duquesne, A. Lemaître, C. Gourdon, and L. Thevenard, Field-free Magnetization Switching by an Acoustic Wave, Phys. Rev. Appl. 11, 014045 (2019).

[18] O. Gladii, D. Halley, Y. Henry, and M. Bailleul, Spin-wave propagation and spin-polarized electron transport in singlecrystal iron films, Phys. Rev. B 96, 174420 (2017).

[19] M. Marangolo, F. Gustavsson, G. M. Guichar, M. Eddrief, J. Varalda, V. H. Etgens, M. Rivoire, F. Gendron, H. Magnan, D. H. Mosca, and J.-M. George, Structural and magnetic anisotropies of Fe/ZnSe(001) thin films, Phys. Rev. B 70, 134404 (2004).

[20] M. Marangolo, F. Gustavsson, M. Eddrief, P. Sainctavit, V. H. Etgens, V. Cros, F. Petroff, J. M. George, P. Bencok, and N. B. Brookes, Magnetism of the Fe/ZnSe(001) Interface, Phys. Rev. Lett. 88, 217202 (2002).

[21] M. Eddrief, Y. Zheng, S. Hidki, B. Rache Salles, J. Milano, V. H. Etgens, and M. Marangolo, Metastable tetragonal structure of $\mathrm{Fe}_{100-x} \mathrm{Ga}_{x}$ epitaxial thin films on ZnSe/GaAs(001) substrate, Phys. Rev. B 84, 161410(R) (2011).

[22] M. Barturen, D. Sander, J. Milano, J. Premper, C. Helman, M. Eddrief, J. Kirschner, and M. Marangolo, Bulklike behavior of magnetoelasticity in epitaxial $\mathrm{Fe}_{1-x} \mathrm{Ga}_{x}$ thin films, Phys. Rev. B 99, 134432 (2019).

[23] S. Adachi, GaAs, AlAs, and $\mathrm{Al}_{x} \mathrm{Ga}_{1-x}$ As: Material parameters for use in research and device applications, J. Appl. Phys. 58, R1 (1985).

[24] D. Royer and E. Dieulesaint, Elastic Waves in Solids II, Advanced Texts in Physics (Springer-Verlag, Berlin Heidelberg, 2000).

[25] F. J. R. Schülein, E. Zallo, P. Atkinson, O. G. Schmidt, R. Trotta, A. Rastelli, A. Wixforth, and H. J. Krenner, Fourier 
synthesis of radiofrequency nanomechanical pulses with different shapes, Nat. Nanotechnol. 10, 512 (2015).

[26] J. Smit and G. Beljers, Ferromagnetic resonance absorption in $\mathrm{BaFe}_{12} \mathrm{O}_{19}$, a highly anisotropic crystal, Philips Res. Rep. 10, 113 (1955).

[27] I. Feng, M. Tachiki, C. Krischer, and M. Levy, Mechanism for the interaction of surface waves with 200-A nickel films, J. Appl. Phys. 53, 177 (1982).

[28] X. Li, D. Labanowski, S. Salahuddin, and C. S. Lynch, Spin wave generation by surface acoustic waves, J. Appl. Phys. 122, 043904 (2017).

[29] Y. Nozaki and S. Yanagisawa, Excitation of ferromagnetic resonance using surface acoustic waves, Electr. Eng. Jpn. 204, 3 (2018).

[30] A. Kamra, H. Keshtgar, P. Yan, and G. E. W. Bauer, Coherent elastic excitation of spin waves, Phys. Rev. B 91, 104409 (2015).

[31] J. Holanda, D. S. Maior, A. Azevedo, and S. M. Rezende, Detecting the phonon spin in magnon-phonon conversion experiments, Nat. Phys. 14, 500 (2018).

[32] It is worthwhile noting that such low frequencies are not probed by Brillouin-light-scattering experiments, which are usually used to measure the magnetic field dependence of magnetization dynamics (i.e., the minimum is smeared out by Brillouin light scattering, hiding the possibility to get subgigahertz resonant MEC.

[33] J.-Y. Duquesne, C. Hepburn, P. Rovillain, and M. Marangolo, Magnetocrystalline and magnetoelastic constants determined by magnetization dynamics under static strain, J. Phys.: Condens. Matter 30, 394002 (2018).

[34] M. F. Lewis and E. Patterson, Acoustic surface wave isolator, Appl. Phys. Lett. 20, 276 (1972).

[35] R. Camley, Nonreciprocal surface waves, Surf. Sci. Rep. 7, 103 (1987).
[36] R. Sasaki, Y. Nii, Y. Iguchi, and Y. Onose, Nonreciprocal propagation of surface acoustic wave in $\mathrm{Ni} / \mathrm{LiNbO}_{3}$, Phys. Rev. B 95, 020407(R) (2017).

[37] We use $2 B_{2}$ here rather than $B_{2}$ (as in Ref. [33]) to conform with the usage in Ref. [8].

[38] T. L. Linnik, A. V. Scherbakov, D. R. Yakovlev, X. Liu, J. K. Furdyna, and M. Bayer, Theory of magnetization precession induced by a picosecond strain pulse in ferromagnetic semiconductor (Ga,Mn)As, Phys. Rev. B 84, 214432 (2011).

[39] L. Dreher, M. Weiler, M. Pernpeintner, H. Huebl, R. Gross, M. S. Brandt, and S. T. B. Goennenwein, Surface acoustic wave driven ferromagnetic resonance in nickel thin films: Theory and experiment, Phys. Rev. B 86, 134415 (2012).

[40] A. K. Ganguly, K. L. Davis, and D. C. Webb, Magnetoelastic surface waves on the (110) plane of highly magnetostrictive cubic crystals, J. Appl. Phys. 49, 759 (1978).

[41] H. Zhou, A. Talbi, N. Tiercelin, and O. Bou Matar, Multilayer magnetostrictive structure based surface acoustic wave devices, Appl. Phys. Lett. 104, 114101 (2014).

[42] P. G. Gowtham, D. Labanowski, and S. Salahuddin, Mechanical back-action of a spin-wave resonance in a magnetoelastic thin film on a surface acoustic wave, Phys. Rev. B 94, 014436 (2016).

[43] A. S. Nowick and B. S. Berry, Anelastic Relaxation in Crystalline Solids (Academic Press, New York and London, 1972).

[44] A. Muñoz-Noval, A. Ordóñez-Fontes, and R. Ranchal, Influence of the sputtering flow regime on the structural properties and magnetic behavior of Fe-Ga thin films ( $\mathrm{Ga} \sim$ 30 at.\%), Phys. Rev. B 93, 214408 (2016). 